\title{
Nonsequential Double Ionization of Atoms in Strong Laser Pulses
}

\author{
J.S. Prauzner-Becheicki ${ }^{a}, \mathrm{~K} . \mathrm{SACHA}^{b}$, B. ECKhardt ${ }^{c}$ \\ AND J. ZAKRZEWSKI ${ }^{b}$ \\ ${ }^{a}$ Faculty of Conservation and Restoration of Works of Art \\ Academy of Fine Arts, Smoleńsk 9, Kraków, Poland \\ ${ }^{b}$ Instytut Fizyki Mariana Smoluchowskiego \\ and Mark Kac Complex Systems Research Center \\ Uniwersytet Jagielloński, Reymonta 4, 30-059 Kraków, Poland \\ ${ }^{c}$ Fachbereich Physik, Philipps-Universität Marburg, 35032 Marburg, Germany
}

It is now possible to produce laser pulses with reproducible pulse shape and controlled carrier envelope phase. It is discussed how that can be explored in double ionisation studies. To this end we solve numerically the Schrödinger equation for a limited dimensionality model which nevertheless treats electron repulsion qualitatively correctly and allows to study correlation effects due to the Coulomb repulsion.

PACS numbers: 32.80.Rm, 32.80.Fb, 03.65.--w, 02.60.Cb, 02.60.-

\section{Introduction}

The existence of significant electron-electron correlations during the ionisation of atoms by intense laser pulses was revealed a quarter of the century ago already [1]. A clear manifestation of those correlations is the celebrated "knee" structure in the double logarithmic plot of the ion yield versus pulsed laser peak intensity [2]. Recent developments of experimental techniques revealed more details of this process by giving access to joint momenta distributions [3-5]. In view of the many degrees of freedom involved and the wide domains that have to be used, full numerical simulations of the process remain a computer demanding task, although some progress may be observed over the years $[6,7]$. Approaches with a reduced computational load include $S$-matrix calculations [8] or dynamical models of lower dimensionality [9-11]. The most popular among those, the so-called aligned electron model (AEM) [9] confines the motion of electrons to one-dimensional tracks along the polarisation axis. 
The accepted picture of the double ionisation process is the rescattering scenario [12]. The electron hit by strong electric field may either leave the atom or come back to the nucleus (if the electric force changes sign due to the oscillatory field time dependence). The first process leads to a direct ionisation. In the second one, the electron brings the energy back, it can be shared with the other electron and the resulting complex may decay either again via a single electron ionisation or with two electrons being simultaneously ejected or the whole rescattering process may be repeated (if external electric field due to the laser pulse is still on).

A couple of years ago two of us proposed a simplified model [13] for a double electron escape from such a complex, a model which links the most probable pathways for the escape with the saddles of the instantaneous electric field formed by the laser and the Coulomb repulsion between the electrons. It turns out that with changing external electric field, the saddles move along lines oriented at an angle of $\pm \pi / 6$ with respect to the polarisation axis. When seeking a reduced dimensionality model for treatment of the ionisation process it seems natural to choose these tracks as the whole configuration space of the problem [14].

In the reduced two-dimensional space the ionisation dynamics may be efficiently simulated [15]. As we have shown, the model correctly reproduces tunnelling and rescattering processes, single and sequential double ionisations. It also correctly mimics the correlated electron escape which is the biggest asset of our model as compared with AEM [9]. The latter overestimates the Coulomb repulsion and suppresses the symmetric escape of electrons.

\section{The model and its basic predictions}

A Hamiltonian for non-relativistic He atom in the new "saddle-track" coordinates is given in atomic units by [14]:

$$
H=\sum_{i=1}^{2}\left(\frac{p_{i}^{2}}{2}-\frac{2}{\left|r_{i}\right|}+\frac{F(t) \sqrt{3}}{2} r_{i}\right)+\frac{1}{\sqrt{\left(r_{1}-r_{2}\right)^{2}+r_{1} r_{2}}},
$$

where $r_{i}$ are electron coordinates along the saddles' lines. The electric field

$$
F(t)=F f(t) \sin (\omega t+\phi),
$$

with $F, f(t), \omega$, and $\phi$ being the peak amplitude, the envelope, the frequency, and the initial phase, respectively.

The Schrödinger equation corresponding to the Hamiltonian (1) is solved on a grid using the well known operator splitting method combined with the fast Fourier transforms (FFT). The potential singularities in (1) are smoothed by the substitution $1 / x \rightarrow 1 / \sqrt{x^{2}+e}$ with $e=0.6$. This leads to a ground state energy of the unperturbed atom $E_{\mathrm{g}}=-2.83$ (found by means of the imaginary time evolution using the same algorithm as for a real time evolution).

The configuration space is divided into regions corresponding to an atom, a singly charged ion, and a doubly charged ion, respectively, following the ideas developed by Dundas et al. [6]. The atom is considered to be singly ionised if one 
electron is far away from and the other is in vicinity of the nucleus. Both electrons close to (or far from) the nucleus correspond to an atom (or a doubly charged ion). Quantitative results depend on the choice of the boundary, of course. But if the boundary conditions are kept fixed, and other parameters like field strength and phase are changed, the model can give valuable information about the relative changes of the signals. Time dependence of different quantities (e.g. ionisation yields) are calculated by evaluating fluxes between different regions [6].

The advantage of that approach is that one can distinguish two physically distinct processes. The sequential double ionisation (SDI) will be defined as a process in which first the boundary for one electron is crossed, then the coordinate of the second electron increases to large values (quantum mechanically that translates into the time sequence for fluxes between different regions). The nonsequential double ionisation (NSDI) will be the process in which we observe a positive flux between the atom and the double ionisation regions directly.

Using in turn a trick proposed by Lein et al. [10] we calculate momenta distributions. The difficulty with the latter is the fact that electrons can travel quite large distances during the laser irradiation. This would require enormous grids to cover a large area of the configuration space. A crucial observation of [10] is to realize that far out the Coulomb force may be neglected and the propagation of the electron may be readily obtained in the velocity gauge as an appropriate phase factor. The details of the procedure may be found elsewhere $[15,16]$, here we shall rather present some exemplary results.

We discuss smooth "sine-squared" laser pulses with envelope $f(t)=$ $\sin ^{2}(\pi t / T)$, where $T$ is the pulse duration. Unless specified explicitly, we assume the carrier phase $\phi=0$.

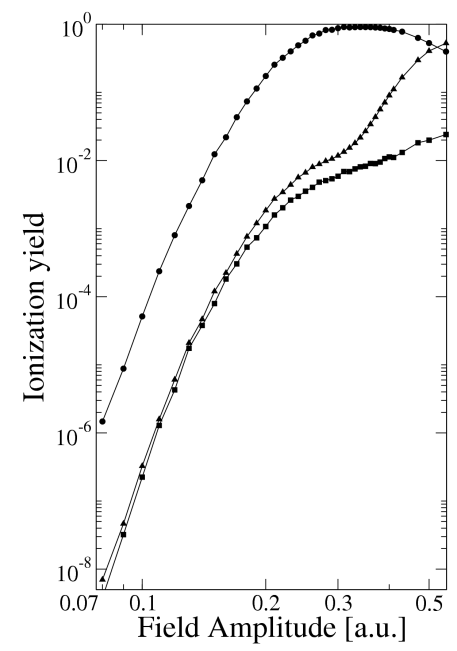

Fig. 1. Single ionisation (circles), sequential double ionisation (triangles); nonsequential double (squares) ionisation as a function of the field amplitude $F_{0}$ for five cycles sine-squared pulses. 
Figure 1 presents total single and double ionisation yields for a pulse of five cycles duration and a fixed phase $\phi=0$. The single ionisation yield shows a typical behaviour reaching almost a $100 \%$ then decreasing for very high intensity due to a significant double ionisation. Double ionisation becomes visible at $F_{0} \approx 0.1$ (for the frequency chosen). Let us observe that the sequential ionisation is always at least slightly more probable than the nonsequential one. For strong field strengths, the nonsequential contribution saturates, but the sequential one increases: this crossover is the famous knee-structure.

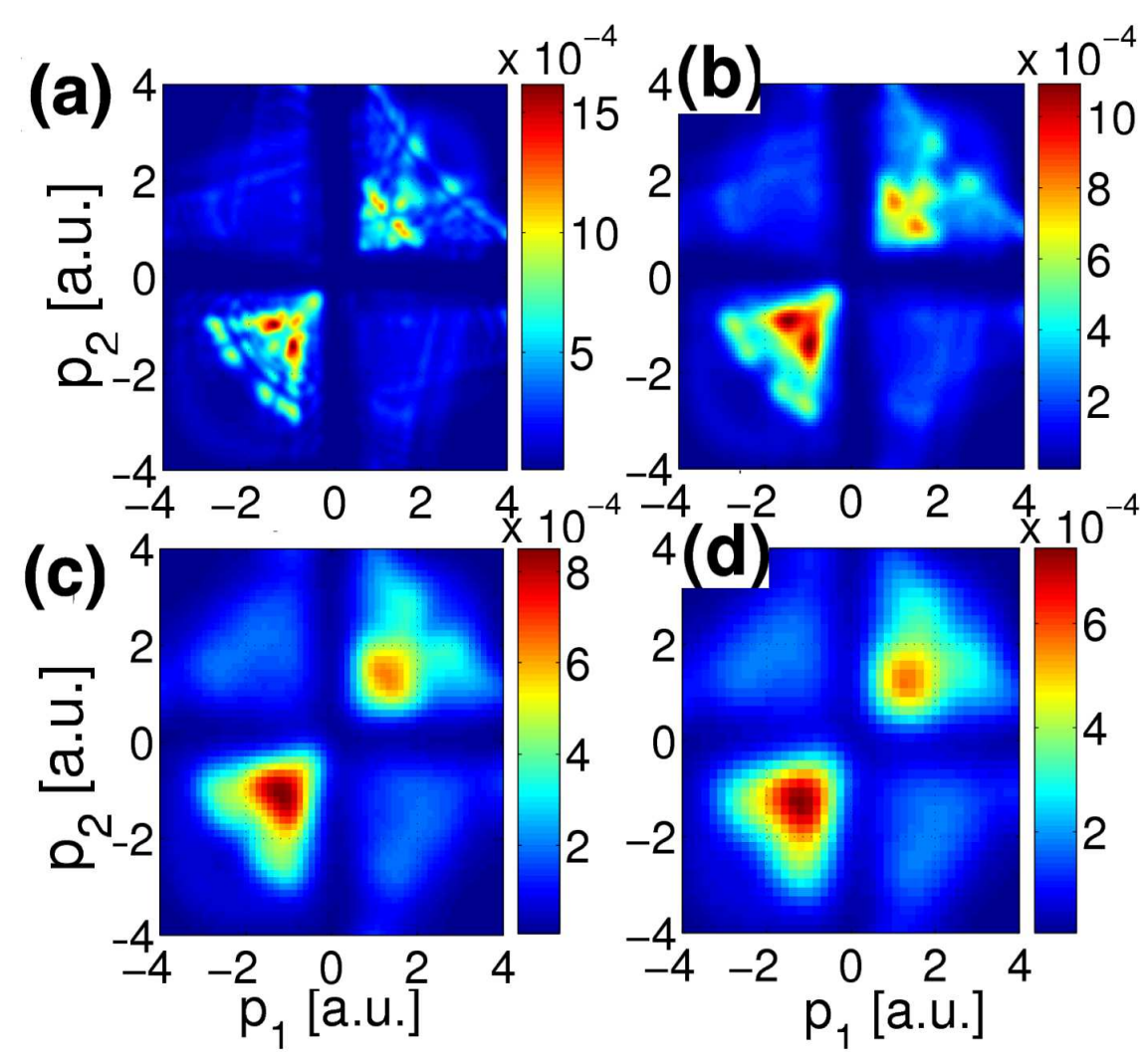

Fig. 2. Electron momenta distributions corresponding to the pulse with the sine-squared envelope, the amplitude $F=0.3$ a.u., the carrier-envelope phase $\phi=0$ and 5 cycles duration. Parts correspond to different resolutions, i.e. (a) 0.07 a.u., (b) 0.16 a.u., (c) 0.3 a.u., and (d) 0.4 a.u.

Let us look now at the momenta distribution for double ionisation. To stay close with experimental reality we smooth the numerical data with Gaussians of different widths. This corresponds to different experimental resolutions, from the best available nowadays $\sigma_{p}=0.07$ a.u. [5] - see part (a) in Fig. 2 to that of the first experiments [4] - part (d). Clearly, a significant portion of electrons escapes 
with symmetric momenta $p_{1} \approx p_{2}$ - the process, let us repeat, underestimated in the aligned electron model [9].

The results are robust with respect to a reasonable change of the field amplitude, $F_{0}$, however, they show a significant dependence on the initial phase $\phi-$ compare Fig. 3. Let us observe that $\phi=0$ and $\phi=\pi$ distributions differ by a

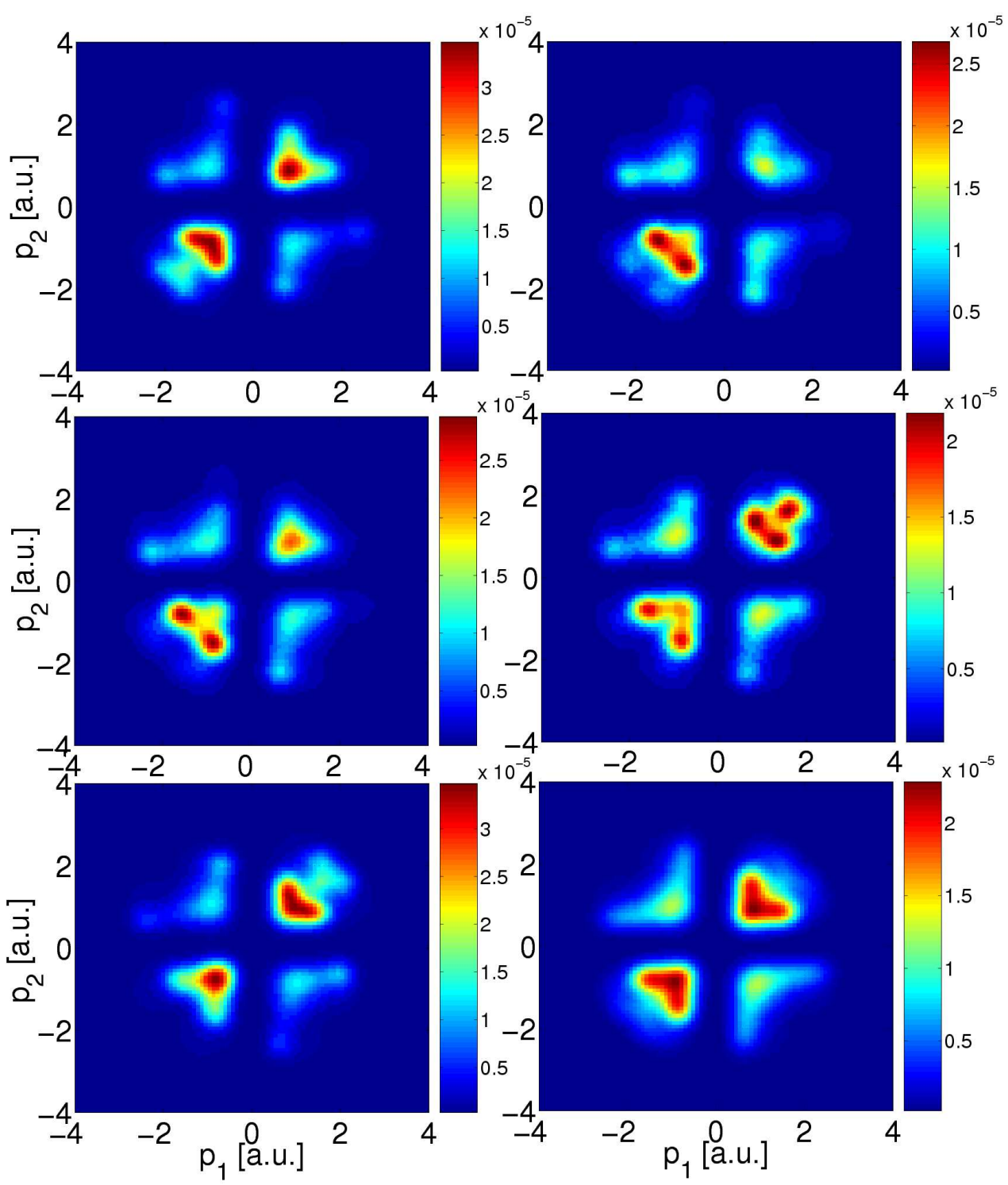

Fig. 3. Electron momenta distributions for $F_{0}=0.2$ and different carrier-envelope phase $\phi=0,0.3 \pi, 0.5 \pi, 0.8 \pi, \pi$ (from top left to bottom left). The bottom right part shows the distribution averaged over a uniform $\phi$ distribution. All parts are plotted with 0.2 a.u. resolution. 


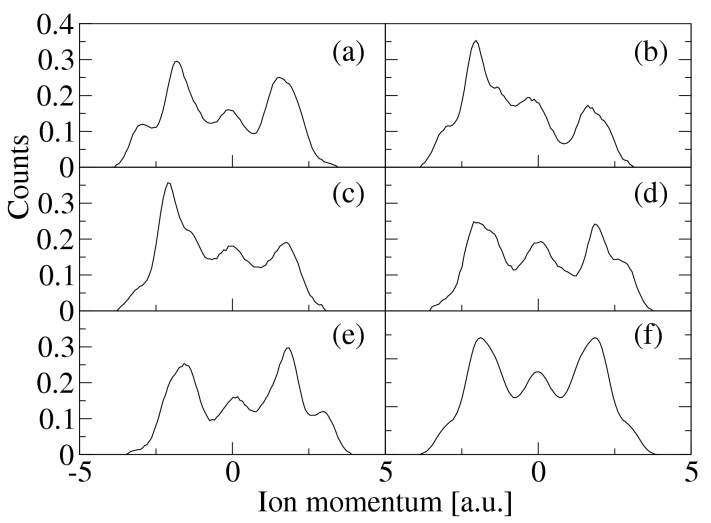

Fig. 4. Ion momenta distributions for $F_{0}=0.2$ and different carrier-envelope phase $\phi=0,0.3 \pi, 0.5 \pi, 0.8 \pi, \pi$ (from top left (a) to bottom left (e)). The bottom right part (f) shows the distribution averaged over a uniform $\phi$ distribution. Let us observe that $\phi=0$ and $\phi=\pi$ distribution differ by a reflection of both momenta axes.

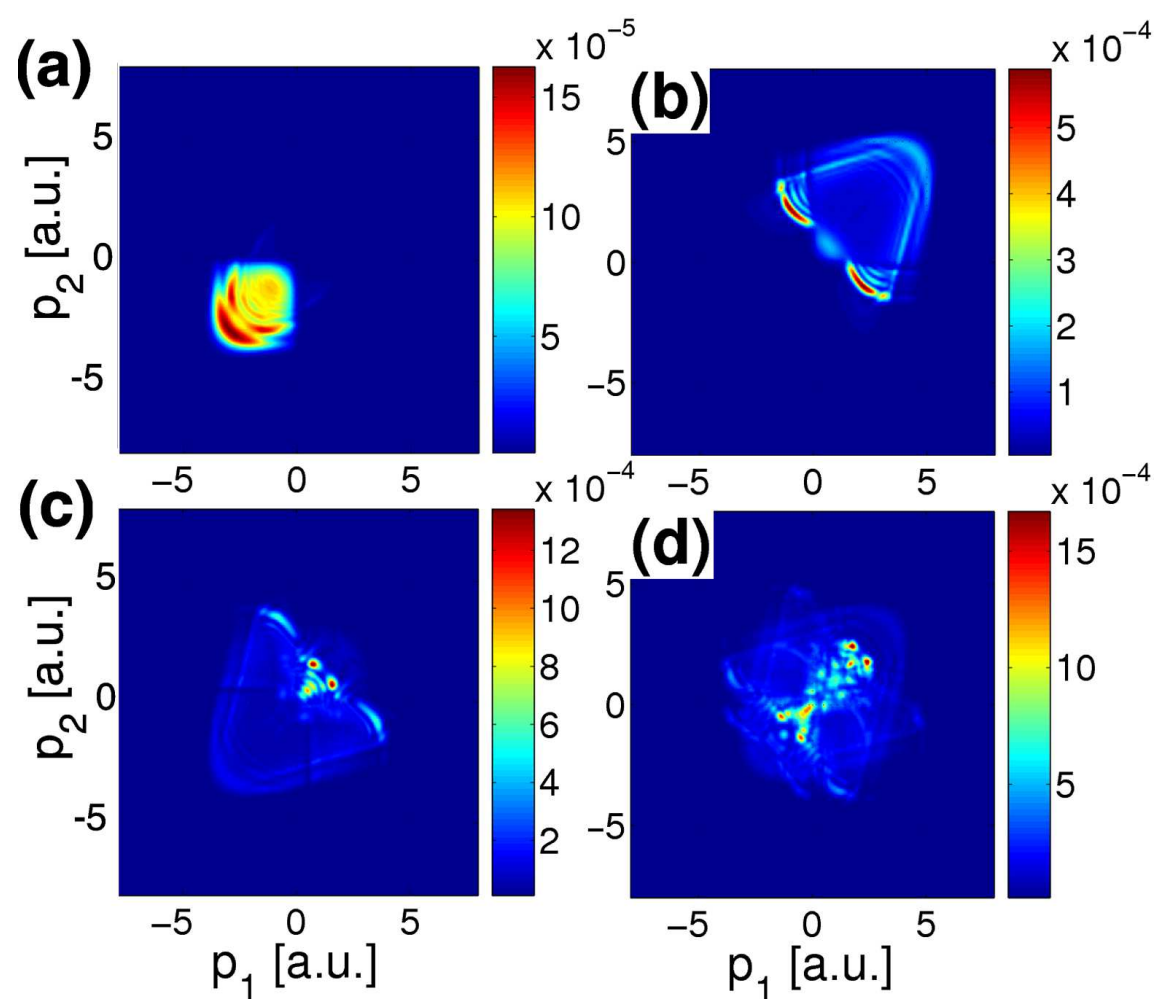

Fig. 5. Electron momenta distributions corresponding to laser pulse with sine-squared envelope, the amplitude $F=0.3$ a.u., the carrier-envelope phase $\phi=0$. Parts correspond to different pulse durations, i.e. (a) 1 cycle, (b) 2 cycles, (c) 3 cycles, and (d) 4 cycles. 
reflection of both momenta axes - this is a general symmetry based behaviour due to the sinusoidal time dependence and the form of the Hamiltonian. The rapid change of the momenta distribution with phase $\phi$ has been suggested as a tool for carrier phase determination $[17,18]$. Judging by our results the signal will not be as pronounced as one might have hoped for.

The electron momenta distributions translate directly into ion recoil momentum distribution since $p_{\text {ion }}=-\sqrt{3} / 2\left(p_{1}+p_{2}\right)$ (with the numerical factor resulting from the $\pi / 3$ angle between the axes of our model (1)). Ion momenta distributions for different carrier-envelope phases are shown in Fig. 4. The distribution averaged over a uniform distribution of carrier envelope phases is of course symmetric and reveals a celebrated double-hump structure (see part (f) in Fig. 4). In addition, our results for $F_{0}=0.2$ reveal the presence of a smaller central minimum corresponding to electrons with antiparallel momenta (coming, e.g. from the sequential process). These correspond to $p_{1} \approx-p_{2}$, i.e. small ion recoil, and are clearly visible in Fig. 3 as light blue island.

The momenta distributions, especially those obtained at the highest resolution, show presence of structures (nodal lines, maxima) resembling interference phenomena. This is due to the fact that several possible paths may lead to double ionisation. If indeed the structures are due to interference, they should be more pronounced and simpler for shorter pulses. Indeed it is the case, as may be observed in Fig. 5. A more detailed analysis of these interference phenomena as well as carrier phase dependence of the observables for very short pulses is in progress.

\section{Conclusions}

The model presented reveals all the key features of the double nonsequential ionisation, i.e. the knee structure in the ionisation yield, the double hump structure in the ion's momentum distribution, and the electrons' momenta distribution showing the signatures of the correlated escape. Both the ion and the electron momenta distributions show a significant dependence on the carrier envelope phase. Momenta distributions for sufficiently short pulses reveal most interesting interference structures. The major advantage of the present model over the well known and studied aligned electron picture is that the latter does not allow for a symmetric simultaneous electron escape due to the overestimation of the Coulomb repulsion. This drawback is absent in our approach.

\section{Acknowledgments}

A significant part of the numerical simulations for this project were done in ICM UW under grant G29-10. The work has been supported by the Deutsche Forschungsgemeinschaft and Marie Curie ToK project COCOS (MTKD-CT-2004-517186). Support by the Polish Government scientific funds (2005-2008) as a research project is acknowledged. 


\section{References}

[1] A. L'Huillier, L.A. Lompre, G. Mainfray, C. Manus, Phys. Rev. Lett. 48, 1814 (1982).

[2] B. Walker, B. Sheehy, L.F. DiMauro, P. Agostini, K.J. Schafer, K.C. Kulander, Phys. Rev. Lett. 73, 1227 (1994).

[3] A. Becker, R. Dörner, R. Moshammer, J. Phys. B 38, S753 (2005).

[4] T. Weber, H. Giessen, M. Weckenbrock, G. Urbasch, A. Staudte, L. Spielberger, O. Jagutzki, V. Mergel, M. Vollmer, R. Dörner, Nature 405, 658 (2000); B. Feuerstein, R. Moshammer, D. Fischer, A. Dorn, C.D. Schröter, J. Deipenwisch, J.R. Crespo Lopez-Urrutia, C. Höhr, P. Neumayer, J. Ullrich, H. Rottke, C. Trump, M. Wittman, G. Korn, W. Sandner, Phys. Rev. Lett. 87, 043003 (2001); R. Moshammer, J. Ullrich, B. Feuerstein, D. Fischer, A. Dorn, C.D. Schröter, J.R. Crespo López-Urrutia, C. Höhr, H. Rottke, C. Trump, M. Wittman, G. Korn, K. Hoffmann, W. Sandner, J. Phys. B 36, L113 (2003).

[5] M. Weckenbrock, A. Becker, A. Staudte, S. Kammer, M. Smolarski, V.R. Bhardwaj, D.M. Rayner, D.M. Villeneuve, P.B. Corkum, R. Dörner, Phys. Rev. Lett. 91, 123004 (2003); M. Weckenbrock, D. Zeidler, A. Staudte, Th. Weber, M. Schöffler, M. Meckel, S. Kammer, M. Smolarski, O. Jagutzki, V.R. Bhardwaj, D.M. Rayner, D.M. Villeneuve, P.B. Corkum, R. Dörner, Phys. Rev. Lett. 92, 213002 (2004).

[6] D. Dundas, K.T. Taylor, J.S. Parker, E.S. Smyth, J. Phys. B 32, L231 (1999).

[7] J.S. Parker, B.J.S. Doherty, K.T. Taylor, K.D. Schultz, C.I. Blaga, L.F. DiMauro, Phys. Rev. Lett. 96, 133001 (2006).

[8] A. Becker, F.H.M. Faisal, Phys. Rev. Lett. 84, 3546 (2000); R. Kopold, W. Becker, H. Rottke, W. Sandner, Phys. Rev. Lett. 85, 3781 (2000).

[9] R. Grobe, J.H. Eberly, Phys. Rev. Lett. 68, 2905 (1992); R. Grobe, J.H. Eberly, Phys. Rev. A 48, 4664 (1993); D. Bauer, Phys. Rev. A 56, 3028 (1997); D.G. Lappas, R. van Leeuwen, J. Phys. B 31, L249 (1998); W.-C. Liu, J.H. Eberly, S.L. Hann, R. Grobe, Phys. Rev. Lett. 83, 521 (1999); S.L. Haan, P.S. Wheeler, R. Panfili, J.H. Eberly, Phys. Rev. A 66, 061402 (2002).

[10] M. Lein, E.K.U. Gross, V. Engel, Phys. Rev. Lett. 85, 4707 (2000).

[11] C. Ruiz, L. Plaja, L. Roso, A. Becker, Phys. Rev. Lett. 96, 053001 (2006).

[12] P.B. Corkum, Phys. Rev. Lett. 71, 1994 (1993); K. Kulander, J. Cooper, K.J. Schafer, Phys. Rev. A 51, 561 (1995).

[13] K. Sacha, B. Eckhardt, Phys. Rev. A 63, 043414 (2001); B. Eckhardt, K. Sacha, Europhys. Lett. 56, 651 (2001).

[14] B. Eckhardt, K. Sacha, J. Phys. B, At. Mol. Phys. 39, 3865 (2006).

[15] J.S. Prauzner-Bechcicki, K. Sacha, B. Eckhardt, J. Zakrzewski, Phys. Rev. Lett. 98, 203002 (2007).

[16] J.S. Prauzner-Bechcicki, K. Sacha, B. Eckhardt, J. Zakrzewski, in preparation.

[17] X. Liu, C. Figueira de Morrison Faria, Phys. Rev. Lett. 92, 133006 (2004).

[18] C. Figueira de Morrison Faria, X. Liu, A. Sanpera, M. Lewenstein, Phys. Rev. A 70, 043406 (2004). 